\title{
Injeção de imidacloprido no controle do pulgão-preto-do-coqueiro
}

\author{
José Inácio L. Moura ${ }^{1 \pm=(\infty)}$, Rosane R. da C. Pereira ${ }^{2(\infty)}$, Lindolfo P. dos Santos Filho ${ }^{3(\mathbb{D}}$, \\ Kátia C. Bispo ${ }^{4 \oplus}$, Raul R. M. Valle ${ }^{3(\infty}$
}

${ }^{1}$ Comissão Executiva do Plano da Lavoura Cacaueira-CEPLAC, Una, BA, Brasil. ' Universidade Federal do Sul da Bahia, Ilhéus, BA, Brasil. ${ }^{3}$ Comissão Executiva do Plano da Lavoura Cacaueira-CEPLAC, Ilhéus, BA, Brasil. ${ }^{4}$ Universidade Estadual de Santa Cruz, Ilhéus, BA, Brasil.

拝=Corresponding author: joseinaciolacerda@gmail.com

Edited by: Ivan C. F. Martins

Received: September 01, 2020. Accepted: November 04, 2020. Published: November 23, 2020.

Trunk injection with Imidacloprid to control the palm aphid

Abstract. The injuries caused by the palm aphid, Cerataphis lataniae, on coconut plants may provoke economic losses. Thus, insecticide injection into the tree can provide protection and control against this pest. Our goal was to evaluate the efficiency of injection of imidacloprid on coconut trees to control the palm aphid. The experiment was carried out at the Lemos Maia experimental station, Ceplac, Una, Bahia, Brazil. The treatments applied were: 1 ) injection of $10 \mathrm{~mL}$ of the commercial product Provado ${ }^{\circledR} 200$ SC (2 g i.a imidacloprid) in the stipe; 2 ) injection of 20 $\mathrm{ml}$ of Provado ${ }^{\circledast} 200 \mathrm{SC}$ diluted in water (1 g i.a imidacloprid) and; 3) control (without insecticide application). The experiment was installed in a completely randomized design, with 15 replications, using leaf number 4 as samples. The parameters analyzed were number of aphids at 3 and 9 months after insecticide application and the number of leaflets/leaves with aphids at 26 months after application. The means were compared by the $\mathrm{F}$ and Tukey test at $5 \%$ probability level. All data were transformed into a log $(x+1)$. The aphid number and the number of leaflets per leaf infested with the aphid was higher in the control treatment. Thus, the application of imidacloprid via injection provided protection to coconut plants to the palm aphid, C. lataniae, until 26 months.

Keywords: Endotherapy, Cerataphis lataniae, insecticide, Arecaceae.

O pulgão-preto-do-coqueiro Cerataphis lataniae Boisduval, 1867 (Hemiptera: Aphididae) possui coloração escura, forma arredondada, com franja branca e diâmetro de 1,5 a 2,0 mm. Ataca coqueiros, suga a seiva de forma contínua, secreta honeydew, que serve de substrato para o desenvolvimento do fungo Capnodium spp. denominado de fumagina. A fumagina forma uma crosta escura sobre o folíolo afetando a fotossíntese do coqueiro e, sua deposição sobre os frutos, desvalorizaos para o consumo in natura (Ferreira et al. 2015). Este afídeo pode provocar abortamento floral, queda de frutos prematuramente (Ferreira \& Michereff Filho 2002) e prefere a parte abaxial dos folíolos das folhas mais novas dos coqueiros (Cocos nucifera L.).

$\mathrm{O}$ ataque de pulgões em coqueiros de porte baixo é facilmente controlado com auxílio de pulverizadores ou atomizadores. Ao contrário, em coqueiros de porte alto, o controle não é satisfatório utilizando-se estes equipamentos. Isto acontece devido a presença de folhas mais velhas que dificultam a adesão de inseticidas nas folhas jovens, que estão dispostas centralmente, local de preferência para colonização pelos pulgões. Desta forma, é inviável aplicar inseticida com atomizador em coqueiros com mais de $10 \mathrm{~m}$ de altura.

A Endoterapia é uma tática fitofarmacêutica que consiste na injeção ou infusão de um produto fitossanitário no tronco de uma árvore ou palmeira, que é translocado através dos tecidos vasculares com objetivo de alcançar partes mais altas do dossel onde pulverizações convencionais não atingiriam. A vantagem é que o composto aplicado é utilizado na sua totalidade quando incorporado ao sistema vascular (Costonis 1981; Wise et al. 2014; Aćimović et al. 2016) e é distribuído por toda a árvore (Kobza et al. 2011). Na endoterapia podem ser veiculados agentes de controle biológico como fungos, bactérias e indutores de resistência (Aćimović et al. 2015; Bahadou et al. 2017). É considerado um método ambientalmente seguro, em virtude da não exposição a organismos não alvos, solo, água, ar, vida selvagem, além de não sofrer efeito negativo das condições climáticas tais como chuva e radiação solar (Aćimović et al. 2019) e minimiza os riscos de contaminação de frutos (Ferreira et al. 2016).

Injeção com imidacloprido é usado em vários países para controlar pragas florestais em cultivos de porte alto ou de espécies arbóreas estabelecidas em áreas urbanas. Nestas áreas, a pulverização não é possível em virtude de problemas com contaminação para a população e seres vivos que ali se inserem. Na Austrália, injeção de imidacloprido foi utilizada no combate ao percevejo bronzeado Thaumastocoris peregrinus Carpintero \& Dellapé, 2006 (Hemiptera: Thaumastocoridae) sobre plantios de eucalipto (Eucalyptus spp.) em áreas urbanas (Noack et al. 2009). Também o percevejo Corythucha ciliata (Say, 1832) (Hemiptera: Tingidae), praga do Platanus occidentalis L. foi eficientemente controlada com injeção de imidacloprido (Chao et al. 2011). Nos Estados Unidos o pulgão Adelges tsugae Annand, 1924 (Hemiptera: Adelgidae), uma severa praga do abeto (Tsuga spp.), tem sido eficientemente controlado com injeção de imidacloprido (Doccola et al. 2007).

Assim, a busca de estratégias de controle de fácil execução, eficientes e de baixo custo operacional e ambientalmente seguro se faz necessária. Desse modo, neste trabalho o objetivo foi avaliar a eficiência de imidacloprido sobre o pulgão-preto quando aplicado em coqueiro através de injeção.

O estudo foi desenvolvido na Estação Experimental Lemos Maia, Ceplac, no município de Una, BA ( $15^{\circ} 17^{\prime} 34^{\prime \prime} \mathrm{S}, 39^{\circ} 04^{\prime} 30^{\prime \prime}$ ) em coqueiros da variedade anão-verde com aproximadamente 20 anos de idade, oito metros de altura e plantados em uma área de 10 ha. As injurias causadas pelos pulgões ao coqueiral estavam restritas a uma área de aproximadamente 4,0 ha.

Para verificar a eficiência de imidacloprido sobre o pulgão utilizouse os seguintes tratamentos: 1 ) injeção com $2 \mathrm{~g}$ do i.a imidacloprido (10 
$\mathrm{mL}$ do produto comercial Provado ${ }^{\circledR} 200 \mathrm{SC}$ puro) no estipe; 2) injeção com $1 \mathrm{~g}$ de i.a imidacloprido $(5 \mathrm{ml}$ de solução do produto comercial Provado $^{\circledR} 200$ SC diluído em $15 \mathrm{~mL}$ de água) e; 3) tratamento controle (sem aplicação de inseticida e sem abertura de orifício). Para aplicação do inseticida utilizou-se uma seringa descartável de $10 \mathrm{~mL}$. Antes, porém, com auxílio de uma furadeira acoplada a uma motosserra, foi feito, em cada planta dos tratamentos 1 e 2, um orifício de $16 \mathrm{~mm}$ de diâmetro e $25 \mathrm{~mm}$ de profundidade, distante $30 \mathrm{~cm}$ do solo onde o inseticida, de coloração branco leitoso, foi introduzido. Esses orifícios não foram fechados. A velocidade de absorção do imidacloprido pela planta foi verificada por meio do tempo de permanência deste inseticida no orifício do estipe. Para tanto, utilizou-se uma seringa de $10 \mathrm{ml}$ para a sucção do líquido presente no orifício, o qual era reaplicado imediatamente. Tal procedimento foi realizado uma vez por semana, até a completa absorção do inseticida, quando apenas líquido incolor foi observado na seringa. $O$ experimento foi conduzido entre $02 / 2016$ a 06/2018 e a aplicação das injeções ocorreu em fevereiro, mês com ocorrência de elevada precipitação.

Os tratamentos foram instalados em um arranjo inteiramente casualizado, com 15 repetições. Como unidade amostral foi utilizada a folha de número 4, que, de acordo com a filotaxia foliar, está mais próxima das folhas centrais, região onde se concentram maior número de pulgões. Os parâmetros analisados foram: número de pulgões no folíolo aos 3 e 9 meses após a aplicação do inseticida e o número de folíolos/folha com pulgões aos 26 meses após a aplicação. No mesmo dia em que se aplicou os tratamentos, um folíolo da folha referência foi retirado de cada planta, os pulgões presentes foram contados e a média de pulgões por folíolo foi de 50,8.

As médias dos parâmetros analisados foram comparadas pelo teste de $\mathrm{F}$ para verificar a existência de diferença entre tratamentos e posteriormente aplicou-se o teste de Tukey ao nível de $5 \%$ de probabilidade como complemento para diferenciação dos tratamentos. Todos os dados foram transformados para log $($ contagem+1), por não apresentarem distribuição normal. Nestas análises se utilizou o procedimento de modelos lineares gerais (Proc GLM) do programa Statiscal Analysis System (SAS Institute 1988).

Houve diferença significativa entre o tratamento controle (sem inseticida) e os tratamentos com inseticida, para as duas variáveis testadas. O número de pulgões no folíolo e o número de folíolos por folha 4 infestado com o afídeo foi maior quando não se aplicou inseticida. As duas concentrações de imidacloprido, veiculadas via injeção, reduziram significativamente o número de pulgões nas folhas dos coqueiros e o número de folíolos por folhas com pulgões, não diferindo entre si. (Tab. 1). Assim proporcionaram um controle eficiente do pulgão quando comparados ao tratamento controle durante o período de avaliação de até 26 meses.

Tabela 1. Médias do número de pulgões por folíolo aos 3 e 6 meses após a aplicação dos tratamentos e número de folíolos por folha 4 com presença de pulgões aos 26 meses após a aplicação de imidacloprido.

\begin{tabular}{lccc}
\hline \multicolumn{1}{c}{ Tratamento } & \multicolumn{2}{c}{$\begin{array}{c}\text { Número de } \\
\text { pulgões/folíolo }\end{array}$} & $\begin{array}{c}\text { Número de folíolos/ } \\
\text { folha } 4 \text { com pulgões }\end{array}$ \\
\cline { 2 - 4 } & 3 meses & 9 meses & 26 meses \\
\hline 1 Injeção de $10 \mathrm{~mL}(2 \mathrm{~g}$ i.a) & $5,5 \mathrm{~b}$ & $0,0 \mathrm{~b}$ & $0,9 \mathrm{~b}$ \\
\hline 1 injeção de $20 \mathrm{ml}$ (1 g i.a) & $4,2 \mathrm{~b}$ & $0,0 \mathrm{~b}$ & $0,8 \mathrm{~b}$ \\
\hline Tratamento controle & $36,1 \mathrm{a}$ & $43,0 \mathrm{a}$ & $84,1 \mathrm{a}$ \\
\hline
\end{tabular}

Teste de Tukey $(p=0,05)$, médias com a mesma letra na coluna não se diferem significativamente. Dados transformados para log (contagem +1$)$.

Observou-se, visualmente, em campo que o imidacloprido (Provado ${ }^{\circledR}$ ) tanto na sua forma concentrada como misturado com água, permaneceu por até 25 dias no orifício do estipe e, uma vez ascendido para a copa, teve ação deletéria sobre o pulgão-preto-do-coqueiro por mais de 2 anos. A demora para ser aspirado pela planta, talvez seja devido sua formulação inadequada para injeção, ou seja, concentração emulsionável e viscosa. Quanto sua longa persistência na copa dos coqueiros, não há relatos na literatura que justifique especificamente essa razão em palmeiras (Aceraceae).

Em cicuta (Tsuga canadensis Carrière), injeções com imidacloprido formuladas especialmente para esse fim, foram injetadas para controlar o pulgão-lanígero-do-pinheiro $A$. tsugae e, por três anos, seu resíduo estava consistentemente acima do valor da $\mathrm{CL}_{50}$ estabelecido para esse pulgão que é de $0,30 \mathrm{\mu g} \mathrm{g}^{-1}$ (Doccola et al. 2012). Conforme esses autores, o longo tempo residual do imidacloprido na copa da cicuta, talvez possa ser explicado pela lenta mobilidade ascendente do caule para a folhagem em virtude de suas propriedades químicas, tais como baixa solubilidade em água ( $\left.510 \mathrm{mg} \mathrm{L}^{-1}\right)$ em comparação a outros inseticidas, inclusive de mesmo grupo químico e, coeficiente de adsorção de carbono (Koc) médio ( 350,32). Tais características poderiam explicar sua mediana adesão no xilema, pois é aventado que o movimento lento do imidacloprido está relacionada a adsorção pela celulose quando na rota apoplástica ou absorção dentro do parênquima axial e radial, no trajeto simplástico. Além dessas propriedades químicas do imidacloprido apresentadas por Doccola et al. 2012, o valor para a constante de Henry é baixo $\left(1,7 \times 10^{-10} \mathrm{~Pa} \mathrm{~m}^{3} \mathrm{~mol}^{-1}\right)$, o que confere menor tendência de volatilização em água, promovendo maior estabilidade da molécula. Tais características podem explicar o resultado desta pesquisa em que a aplicação do imidacloprido reduziu o número de afídeos atacando as plantas por mais de 2 anos.

Injeção com imidacloprido para controlar o pulgão preto do coqueiro é uma abordagem química em parte sustentável, pois não afeta os organismos que vivem no solo, não polui a água e o ar atmosférico, pois não ocorre deriva e, aparentemente, não é deletério aos insetos que congregam as inflorescências do coqueiro quando em ântese. No entanto, a exemplo de outros cultivos, o perfil residual nos frutos, pólen e néctar deve ser analisado, pois é um complemento na endoterapia. Ferreira et al. (2016) verificaram que não houve contaminação de cachos de coco e coco seco por produtos fitossanitários, incluindo o imidacloprido, aplicados em coqueiros, via endoterapia, até 120 dias após a aplicação. Wise et al. (2014) determinaram o perfil residual em frutos de maçã oriundas de macieiras (Malus domestica, Borkh) que receberam injeções de imidacloprido. Os resíduos detectados, em dias após injeções (DAI), foram: 29 DAl, 0,05 ppm e aos 47 DAl, 0,04 ppm. Conforme estes autores, os limites máximos de resíduo (LMR) estabelecido pela agência ambiental dos Estados Unidos para o imidacloprido em frutos de macieira é de 0,5 ppm (USEPA 1997; citado por Wise et al. 2014). Quanto ao perfil residual de imidacloprido no pólen e néctar das macieiras, não foi identificado nenhum resíduo durante a primavera. No entanto, ao final da primavera foi detectado $0,39 \mathrm{ng} \mathrm{g}^{-1}$. O limiar estabelecido por essa mesma agência ambiental dos EUA para as abelhas é de $25 \mathrm{ng} \mathrm{g}^{-1}$ (Coslor et al. 2018).

Injeções em palmeiras é uma tática usual que vem sendo praticada há mais de 40 anos em coqueiros, dendezeiros (Elaeis guineenses J.) e tamareiras (Phoenix dactylifera L.) nos continentes Africano, Asiático e Americano e, nunca foi relatado problemas biomecânicos e/ou patologias em decorrência a essa prática (Ferry \& Gomez 2014). Mais recentemente, injeções com neonicotinoides e ivermectinas tem sido aplicada no controle de Rhynchophorus ferrugineus (Olivier, 1790) (Coleoptera: Curculionidae), a praga das palmeiras mais destrutiva e invasiva do mundo (Dembilio et al. 2014). Vários métodos de controle já foram testados, incluindo pulverizações tópicas na região da coroa foliar das palmeiras, métodos biológicos e uso de feromônio na coleta massal (Faleiro et al. 2012; Giblin-Davis et al. 2013; Rochat et al. 2017). No entanto, injeção tem sido o método mais comum para controlar R. ferrugineus e, conforme Chihaoui-Meridja et al. (2020) além de eficiente, não tem efeito adverso sobre espécies não alvos, seres humanos e o meio ambiente. Injeções em palmeiras-das-canárias (Phoenix canariensis Chabaud) visando determinar a eficiência de tiametoxam e benzoato de emamectina revelaram que tiametoxam causou mortalidade de 55,7\% em adultos $R$. ferrugineus, 97,3\% em pupas e $96,6 \%$ em larvas. No entanto, tiametoxam teve ação preventiva e não curativa. Por sua vez, benzoato de emamectina teve eficiência próxima de $100 \%$ na mortalidade de adultos pupas e larvas tanto no âmbito preventivo como curativo (Chihaoui-Meridja et al. 2020).

As monocotiledôneas, especialmente as palmeiras, não compartimentalizam seus ferimentos segundo o modelo CODIT, como estabelecido por Shigo (1977). Embora não formem barreiras (calos) nas partes feridas para evitar estresse por evaporação e penetração 
de fungos xilófagos a exemplo das dicotiledôneas, não significa que as palmeiras não sejam capazes de "curar" seus ferimentos, pois se não tivesse essa capacidade, certamente nunca teriam surgido no mundo vegetal (Ferry \& Gomez 2014). Apesar das palmeiras não terem cambio vascular e crescimento secundários a exemplo das dicotiledôneas, as áreas danificadas das palmeiras são rapidamente isoladas por células do parênquima para vascular, que secretam tilose, compostos fenólicos e um gel ácido contendo pectina (Thomas \& Franceschi 2013). Cabe ressaltar que as palmeiras por não terem cambio e felógeno, constitui uma vantagem comparativamente as dicotiledôneas, pois tais tecidos oferecerem menos resistência a danos por microrganismos e são mais sensíveis a problemas de fitotoxidade. Outro aspecto a ser considerado, diz respeito ao centro das árvores, cerne, e ao centro do estipe nas palmeiras. O cerne é uma espécie de coração da árvore e, essa região não tem mais tecido vivo, pois à medida que as células do alburno decaem e morrem vão sendo incorporadas ao cerne. Em razão disso, por não terem mecanismos ativos de defesa, as dicotiledôneas não resistem a invasão de microrganismos. Já a parte central das palmeiras não é composto de tecido morto, mas de feixes vasculares e tecidos parenquimáticos que permanecem indefinidamente vivos e, por essa razão, são capazes de empregar mecanismos ativos para reagirem a um dano. Esta diferença explica por que a profundidade da injeção é menos importante nas palmeiras do que nas árvores (Ferry \& Gomez 2014).

Foi observado que os coqueiros no tratamento controle e aqueles que não compunham a unidade experimental, mostravam-se muito atacados pela cochonilha-transparente-do-coqueiro Aspidiotus destructor Signoret, 1869 (Hemiptera: Diaspididae) e a mosca-brancado-coqueiro Aleurodicus pseudugesii Martin, 2008 (Hemiptera: Aleyrodidade). Assim, pode-se inferir, que com uma única injeção de imidacloprido é possível reduzir a densidade populacional de outros insetos sugadores do coqueiro, além do pulgão-preto-do-coqueiro.

Desta forma, a aplicação de imidacloprido, via injeção, proporcionou redução da densidade populacional do pulgão $C$. lataniae em coqueiros por até 26 meses. Novos testes, com avaliações semestrais, devem ser conduzidos para verificar o perfil residual do produto fitossanitário nos frutos, haja vista a importância destes dados para a segurança alimentar. Também deverão ser realizadas avaliações da população de sugadores e da taxa de translocação do imidacloprido para as folhas.

\section{Agradecimentos}

A José Alves Duarte pelo apoio no campo.

\section{Contribuição dos Autores}

J.I.L.M.: conceptualização, delineamento, implantação da pesquisa, redação e edição do manuscrito; R.R.C.P.: redação e edição do manuscrito; L.P.S.: análise dos dados; K.C.: conceitualização e delineamento; R.R.M.V.: conceitualização e delineamento.

\section{Referências}

Aćimović, S. G.; Zeng, Q.; Mcghee, G. C.; Sundin, G. W.; Wise, J. C. (2015) Control of fire blight (Erwinia amylovora) on apple trees with trunkinjected plant resistance inducers and antibiotics and assessment of induction of pathogenesis-related protein genes. Frontiers in Plant Science, 6(16): 1-10. doi: 10.3389/fpls.2015.00016

Aćimović, S. G.; Cregg, B. M.; Sundin, G. W.; Wise, J. C. (2016) Comparison of drill-and needle-based tree injection technologies in healing of trunk ports on apple trees. Urban Forestry \& Urban Greening, 19(1): 151-157. doi: 10.1016/j.ufug.2016.07.003

Aćimović, S. G.; Martin, D. K. H; Turcotte, R. M.; Meredith, C. L.; Munck, I. A. (2019) Choosing an adequate pesticide delivery system for managing pathegens with difficult biologies: case studies on Diplodia cortícola, Venturia inaequalis and Erwinia amylovora. In: Topolovec-Pintaric, S. (Ed), Plant Pathology and Management of Plant Diseases, pp. 1-36. Zagreb: University of Zagreb. doi: 10.5772/ intechopen.87956
Bahadou, S. A.; Ouijja, A.; Boukhari, M. A.; Tahiri, A. (2017) Development of field strategies for fire blight control integrating biocontrol agents and plant defense activators in Marocco. Journal of Plant Pathology, 99: 51-58. doi: 10.4454/jpp.v99i0.3909

Chao, L.; Peng, L.; Yang, Z; Chuan-Ren, L.; Wei, Z.; Wen-Sheng, X. (2011) Study on effects and residue effects of root-irrigating and trunkinjecting of imidacloprid and acephate for control of Corythucha ciliate (SAY). Hubei: Hubei Agricultural Sciences. https://en.cnki. com.cn/Article_en/CJFDTotal-HBNY201114022.htm. Access on: 10.xi.2020

Chihaoui-Meridja, S.; Harbi, A.; Abbes, K.; Chaabane, H.; La Pergola, A.; Chermiti, B.; Suma, P. (2020) Systematicity, persistence and efficacy of selected insecticides used in endotherapy to control the red palm weevil Rhynchophorus ferrugineus (Olivier, 1790) on Phoenix canariensis. Phytoparasitica, 48: 75-85. doi: 10.1007/s12600-01900776-5

Coslor, C.; Vandervoort, C.; Wise, J. C. (2018) Insecticide dose and seasonal timing of trunk injection in apples influence efficacy and residues in nectar and plant parts. Pest Management Science, 75 : 1453-1463. doi: 10.1002/ps.5268

Costonis, A. C. (1981) Tree injection: Perspective macro-injection/ micro-injection. Journal of Arboriculture, 7(10): 275-278.

Doccola, J. J.; Bristol, E. J.; Sifleet, S. D.; Lojko, J.; Wild, P. M. (2007) Efficacy and duration of trunk-injected imidacloprid in the management of hemlock woolly adelgid (Adelges tsugae). Arboriculture \& Urban Forestry, 33(1): 12-21.

Doccola, J. J.; Hascher, W.; Aiken, J. J.; Wild, P. M. (2012) Treatment strategies using imidacloprid in hemlock woolly adelgid (Adelges tsugae Annand) infested eastern hemlock (Tsuga canadensis Carrière) trees. Arboricult Urban Forestry, 38(2): 41-49.

Dembilio, Ó.; Riba, J. M.; Gamón, M.; Jacas, J. A. (2014) Mobility and efficacy of abamectin and imidacloprid against Rhynchophorus ferrugineus in Phoenix canariensis by different application methods. Pest Management Science, 71(8): 1091-1098. doi: 10.1002/ps.3889

Faleiro, J. R., Abdallah, A. B., El-Bellaj, M., Al-Ajlan, A. M.; Oihabi, A. (2012) Threat of the red palm weevil, Rhynchophorus ferrugineus (Olivier) to date palm plantations in North Africa. Arab Journal of Plant Protection, 30(2): 274-280.

Ferreira, J. M. S.; Michereff Filho, M. (2002) Produção integrada de coco: Práticas Fitossanitárias. Embrapa. Aracaju.

Ferreira, J. M. S.; Teodoro, A. V.; Negrisoli Júnior, A. S.; Guzzo, E. C. (2015) Aspectos biológicos e manejo da cochonilha transparente Aspidiotus destructor S. e do pulgão preto Cerataphis lataniae B. em coqueiro. Comunicado Técnico, 177, Embrapa: Aracaju.

Ferreira, J. A.; Ferreira, J. M. S.; Talamini, V.; Facco, J. F.; Rizzetti, T. M.; Prestes, O. D.; Adaime, M. B.; Zanella, R.; Bottoli, C. B. G. (2016) Determination of pesticides in coconut (Cocos nucifer Linn.) water and pulp using modified QuEChERS and LC-MS/MS. Food Chemistry, 213(15): 616-624. doi: 10.1016/j.foodchem.2016.06.114

Ferry, M.; Gomez, S. (2014) Assessment of risks and potential of injection techniques in integrated programs to eradicate the red palm weevil: review and new perspectives. Fruits, 69(2): 143-157. doi: 10.1051/fruits/2014005

Giblin-Davis, R. M.; Faleiro, J. R.; Jacas, J. A.; Peña, J. E.; Vidyasagar, P. S. P. V. (2013) Biology and management of the red palm weevil, Rhynchophorus ferrugineus. In: Peña, J. E. (Ed.), Potential invasive pests of agricultural crops, pp. 1-34. Florida: CABI. doi:10.1079/9781845938291.0000

Kobza, M.; Juhásová, G.; Adamcíková, K.; Onrusková, E. (2011) Tree injection in the management of Horse-Chestnut leaf miner. Cameraria ohridella (Lepidoptera: Gracillariidae). Gesunde Pflanzen, 62: 139-143. doi:org/10.1007/s10343-011-0236-z

Noack, A. E.; Kaapro, J.; Bartimote-Aufflick, K.; Mansfield, S.; Rose, H. A. (2009) Efficacy of Imidacloprid in the control of Thaumastocoris peregrinus on Eucalyptus scoparia in Sydney, Australia. Arboriculture \& Urban Forestry, 35(4): 192-196.

Rochat, D.; Dembilio, Ó.; Jaques, A. J.; Suma, P.; La Pergola, A.; Hamidi, R.; Kontodimas, D.; Soroker, V. (2017) Rhynchophorus Ferrugineus: taxonomy, distribution, biology, and life cycle. In: Soroker \& Colazza 
(Eds.), Handbook of major palm pests: Biology and management, pp. 69-104. West Sussex: John Wiley \& Sons Ltda.

SAS Institute (1988) SAS/STAT User's Guide. Cary: SAS Institute Inc. https://www.sas.com/pt_br/home.html

Shigo A. L.; Marx H. G. (1977) Compartmentalization of decay in trees, Washington: U.S. Department of Agriculture. https://www. fs.fed.us/nrs/pubs/jrnl/1985/ne_1985_shigo_001.pdf. Access on: 10.xi.2020.

Thomas, R.; Franceschi, D. (2013) Palm stem anatomy and computeraided identification: the Coryphoideae (Arecaceae). American Journal of Botany, 100(2): 289-313. doi: 10.3732/ajb.1200242

Wise, J. C.; Vanwoerkom, A. H.; Aćimović, S. G.; Sundin, G. W.; Cregg, B. M.; Vandervoort, C. (2014) Trunk Injection: a discriminating delivering system for horticulture crop IPM. Entomology, Ornithology \& Herpetology, 3(2): 1-7. doi: 10.4172/21610983.1000126 